\title{
Heat Transfer in a Medium in Which Many Small Particles Are Embedded
}

\author{
A. G. Ramm* \\ Department of Mathematics \\ Kansas State University, Manhattan, KS 66506-2602, USA
}

\begin{abstract}
The heat equation is considered in the complex system consisting of many small bodies (particles) embedded in a given material. On the surfaces of the small bodies a Newtontype boundary condition is imposed. An equation for the limiting field is derived when the characteristic size $a$ of the small bodies tends to zero, their total number $\mathcal{N}(a)$ tends to infinity at a suitable rate, and the distance $d=d(a)$ between neighboring small bodies tends to zero $a<<d$. No periodicity is assumed about the distribution of the small bodies.
\end{abstract}

Keywords and phrases: heat transfer, many-body problem

Mathematics Subject Classification: 35K20, 35J15, 80M40, 80A20

\section{Introduction}

Let many small bodies (particles) $\mathcal{D}_{m}, 1 \leq m \leq M$, be distributed in a bounded domain $\mathcal{D} \subset \mathbb{R}^{3}$, $\operatorname{diam} \mathcal{D}_{m}=2 a$. The small bodies are distributed according to the law

$$
\mathcal{N}(\Delta)=\frac{1}{a^{2-\kappa}} \int_{\Delta} N(x) d x[1+o(1)], a \rightarrow 0 .
$$

Here $\Delta \subset \mathcal{D}$ is an arbitrary open subdomain of $\mathcal{D}, \kappa \in(0,1)$ is a constant, $N(x) \geq 0$ is a continuous function, and $\mathcal{N}(\Delta)$ is the number of the small bodies $\mathcal{D}_{m}$ in $\Delta$. Let us assume that the boundaries $S_{m}$ of the bodies $\mathcal{D}_{m}$ are $C^{2}$-smooth.

The heat equation can be stated as follows:

$$
\begin{gathered}
u_{t}=\nabla^{2} u+f(x) \text { in } \mathbb{R}^{3} \backslash \bigcup_{m=1}^{M} \mathcal{D}_{m}:=\Omega,\left.u\right|_{t=0}=0, \\
u_{N}=\zeta_{m} u \text { on } \mathcal{S}_{m}, 1 \leq m \leq M .
\end{gathered}
$$

\footnotetext{
${ }^{*}$ Corresponding author. E-mail: ramm@math.ksu.edu
} 
Here $N$ is the outer unit normal to $\mathcal{S}:=\bigcup_{m=1}^{M} \mathcal{S}_{m}, \zeta_{m}=\frac{h\left(x_{m}\right)}{a^{\kappa}}, x_{m} \in \mathcal{D}_{m}, 1 \leq m \leq M$, where $h(x)$ is a continuous function in $\mathcal{D}$.

The aim of this paper is to establish a method for solving both theoretically and numerically the many-body heat transfer problem, and to derive an equation for the effective (self-consistent) field in the medium in which many small bodies (particles) are embedded.

There is a large literature on homogenization theory which deals with similar problems, see [1]-[4] and the literature therein. In most cases it is assumed in the homogenization literature that the coefficients of the equations are periodic, and there is a small parameter in these coefficients, that the related problems in a "periodic cell" have discrete spectrum and are selfadjoint, that the elliptic estimates, such as Rellich and Poincare inequalities hold, etc. Our method, which was used in [5]-[19], differs in many respects from the homogenization theory methods developed in the literature: periodicity assumption is not used, the spectrum of the related elliptic problems that we use is continuous, the problems may be non-selfadjoint, and our justification of the limiting equation for the effective field is based on a new technique, namely, on convergence results for collocation methods (see, [7]). The published homogenization techniques are not directly applicable to our problem because of the lack of periodicity.

The main results of this paper are: 1) Derivation of the linear algebraic system (25) for finding the Laplace transform of the solution to problem (2)-(3),

2) Derivation of the equation (29) for the limiting effective field in the medium when the size of the small bodies tends to zero while the total number of these bodies tends to infinity according to the distribution law (1),

3) Derivation of the formula (31) for the average temperature in the limiting medium.

In Section 2 a derivation of these results is given, and in Section 3 proofs of some lemmas are given.

\section{Derivation of the equation for the limiting effective field}

Denote

$$
\mathcal{U}:=\mathcal{U}(x, \lambda)=\int_{0}^{\infty} e^{-\lambda t} u(x, t) d t
$$

Then, (1.2) - (1.3) imply

$$
\begin{gathered}
-\nabla^{2} \mathcal{U}+\lambda \mathcal{U}=\lambda^{-1} f(x) \text { in } \Omega, \\
\mathcal{U}_{N}=\zeta_{m} \mathcal{U} \text { on } \mathcal{S}_{m}, 1 \leq m \leq M
\end{gathered}
$$

Let

$$
g(x, y):=g(x, y, \lambda)=\frac{e^{-\sqrt{\lambda}|x-y|}}{4 \pi|x-y|}, \quad \frac{1}{\lambda} \int_{\mathbb{R}^{3}} g(x, y) f(y) d y=F(x, \lambda) .
$$

Look for the solution to $(2.1)-(2.2)$ of the form

$$
\mathcal{U}(x, \lambda)=F(x, \lambda)+\sum_{m=1}^{M} \int_{\mathcal{S}_{m}} g(x, s) \sigma_{m}(s) d s, \quad \mathcal{U}(x, \lambda):=\mathcal{U}(x):=\mathcal{U},
$$

where $\sigma_{m}$ are unknown and should be found from the boundary conditions (2.2). Equation (2.1) is satisfied by $\mathcal{U}$ of the form (2.4) for any $\sigma_{m}$. To satisfy (2.2) one has to solve equation

$$
\frac{\partial \mathcal{U}_{e}(x)}{\partial N}+\frac{A_{m} \sigma_{m}-\sigma_{m}}{2}-\zeta_{m} \mathcal{U}_{e}-\zeta_{m} T_{m} \sigma_{m}=0 \text { on } \mathcal{S}_{m}, 1 \leq m \leq M .
$$

Here

$$
\mathcal{U}_{e}(x):=\mathcal{U}_{e, m}(x):=\mathcal{U}(x)-\int_{\mathcal{S}_{m}} g(x, s) \sigma_{m}(s) d s,
$$




$$
T_{m} \sigma_{m}=\int_{\mathcal{S}_{m}} g\left(s, s^{\prime}\right) \sigma_{m}\left(s^{\prime}\right) d s^{\prime}, A_{m} \sigma_{m}=2 \int_{\mathcal{S}_{m}} \frac{\partial g\left(s, s^{\prime}\right)}{\partial N_{S}} \sigma_{m}\left(s^{\prime}\right) d s^{\prime},
$$

and the known formula for the outer limiting value on $\mathcal{S}_{m}$ of the normal derivative of a simple layer potential was used. We now apply the ideas and methods for solving many-body scattering problems developed in [5] - [7].

Let us call $\mathcal{U}_{e, m}$ the effective (self-consistent) value of $\mathcal{U}$, acting on $m$-th body. As $a \rightarrow 0$, the dependence on $m$ disappears, since

$$
\int_{\mathcal{S}_{m}} g(x, s) \sigma_{m}(s) d s \rightarrow 0 \text { as } a \rightarrow 0
$$

One has

$$
\mathcal{U}(x, \lambda)=F(x, \lambda)+\sum_{m=1}^{M} g\left(x, x_{m}\right) Q_{m}+\mathcal{J}_{2}, \quad x_{m} \in \mathcal{D}_{m},
$$

where

$$
Q_{m}:=\int_{\mathcal{S}_{m}} \sigma_{m}(s) d s
$$

and

$$
\mathcal{J}_{2}:=\sum_{m=1}^{M} \int_{\mathcal{S}_{m}}\left[g\left(x, s^{\prime}\right)-g\left(x, x_{m}\right)\right] \sigma_{m}\left(s^{\prime}\right) d s^{\prime}, \mathcal{J}_{1}:=\sum_{m=1}^{M} g\left(x, x_{m}\right) Q_{m} .
$$

We prove (in Section 3) that

$$
\left|\mathcal{J}_{2}\right|<<\left|\mathcal{J}_{1}\right| \text { as } a \rightarrow 0
$$

provided that

$$
\lim _{a \rightarrow 0} \frac{a}{d(a)}=0 .
$$

where $d(a)=d$ is the minimal distance between neighboring particles.

If (2.10) holds, then problem (2.1) - (2.2) is solved asymptotically by the formula

$$
\mathcal{U}(x, \lambda)=F(x, \lambda)+\sum_{m=1}^{M} g\left(x, x_{m}\right) Q_{m}, a \rightarrow 0,
$$

provided that asymptotic formulas for $Q_{m}$, as $a \rightarrow 0$, are found. To find formulas for $Q_{m}$, let us integrate (2.5) over $\mathcal{S}_{m}$ and estimate the order of the terms in the resulting equation as $a \rightarrow 0$. We get

$$
\int_{\mathcal{S}_{m}} \frac{\partial \mathcal{U}_{e}}{\partial N} d s=\int_{\mathcal{D}_{m}} \nabla^{2} \mathcal{U}_{e} d x=O\left(a^{3}\right) .
$$

Here we assumed that $\left|\nabla^{2} \mathcal{U}_{e}\right|=O(1), a \rightarrow 0$. This assumption will be justified in Section 2 .

$$
\int_{\mathcal{S}_{m}} \frac{A_{m} \sigma_{m}-\sigma_{m}}{2} d s=-Q_{m}[1+o(1)], \quad a \rightarrow 0 .
$$

This relation is justified in Section 2. Furthermore,

$$
-\zeta_{m} \int_{\mathcal{S}_{m}} \mathcal{U}_{e} d s=-\zeta_{m}\left|\mathcal{S}_{m}\right| \mathcal{U}_{e}\left(x_{m}\right)=O\left(a^{2-\kappa}\right), a \rightarrow 0
$$

where

$$
\left|\mathcal{S}_{m}\right|=O\left(a^{2}\right)
$$


is the surface area of $\mathcal{S}_{m}$. Finally,

$$
\begin{gathered}
-\zeta_{m} \int_{\mathcal{S}_{m}} d s \int_{\mathcal{S}_{m}} g\left(s, s^{\prime}\right) \sigma_{m}\left(s^{\prime}\right) d s^{\prime}=-\zeta_{m} \int_{\mathcal{S}_{m}} d s^{\prime} \sigma_{m}\left(s^{\prime}\right) \int_{\mathcal{S}_{m}} d s g\left(s, s^{\prime}\right) \\
=Q_{m} O\left(a^{1-\kappa}\right), \quad a \rightarrow 0 .
\end{gathered}
$$

Thus, the main term of the asymptotics of $Q_{m}$ is

$$
Q_{m}=-\zeta_{m}\left|\mathcal{S}_{m}\right| \mathcal{U}_{e}\left(x_{m}\right)
$$

Formulas (2.17) and (2.12) yield

$$
\mathcal{U}(x, \lambda)=F(x, \lambda)-\sum_{m=1}^{M} g\left(x, x_{m}\right) \zeta_{m}\left|\mathcal{S}_{m}\right| \mathcal{U}_{e}\left(x_{m}, \lambda\right),
$$

and

$$
\mathcal{U}_{e}\left(x_{m}, \lambda\right)=F\left(x_{m}, \lambda\right)-\sum_{m^{\prime} \neq m, m^{\prime}=1}^{M} g\left(x_{m}, x_{m^{\prime}}\right) \zeta_{m^{\prime}}\left|\mathcal{S}_{m^{\prime}}\right| \mathcal{U}_{e}\left(x_{m^{\prime}}, \lambda\right) .
$$

Denote

$$
\begin{gathered}
\mathcal{U}_{e}\left(x_{m}, \lambda\right):=\mathcal{U}_{m}, \\
F\left(x_{m}, \lambda\right):=F_{m}, \quad g\left(x_{m}, x_{m^{\prime}}\right):=g_{m m^{\prime}},
\end{gathered}
$$

and write (2.19) as a linear algebraic system

$$
\mathcal{U}_{m}=F_{m}-a^{2-\kappa} \sum_{m^{\prime} \neq m} g_{m m^{\prime}} h_{m^{\prime}} c_{m^{\prime}} \mathcal{U}_{m^{\prime}}, \quad 1 \leq m \leq M,
$$

where

$$
h_{m^{\prime}}=h\left(x_{m^{\prime}}\right), \quad \zeta_{m^{\prime}}=\frac{h_{m^{\prime}}}{a^{\kappa}}, \quad c_{m^{\prime}}:=\left|S_{m^{\prime}}\right| a^{-2} .
$$

Consider a partition of the bounded domain $\mathcal{D}$, in which the small bodies are distributed, into a union of $P<<M$ small nonintersecting cubes $\Delta_{p}, 1 \leq p \leq P$, of side $b>>d, b=b(a) \rightarrow 0$ as $a \rightarrow 0$. Let $x_{p} \in \Delta_{p},\left|\Delta_{p}\right|=$ volume of $\Delta_{p}$. One has

$$
\begin{gathered}
a^{2-\kappa} \sum_{m^{\prime}=1, m^{\prime} \neq m}^{M} g_{m m^{\prime}} h_{m^{\prime}} c_{m^{\prime}} \mathcal{U}_{m^{\prime}}=a^{2-\kappa} \sum_{p^{\prime}=1, p^{\prime} \neq p}^{P} g_{p p^{\prime}} h_{p^{\prime}} c_{p^{\prime}} \mathcal{U}_{p^{\prime}} \sum_{x_{m^{\prime}} \in \Delta_{p^{\prime}}} 1= \\
=\sum_{p^{\prime} \neq p} g_{p p^{\prime}} h_{p^{\prime}} c_{p^{\prime}} \mathcal{U}_{p^{\prime}} N\left(x_{p^{\prime}}\right)\left|\Delta_{p^{\prime}}\right|[1+o(1)], \quad a \rightarrow 0 .
\end{gathered}
$$

Thus, (2.20) yields

$$
\mathcal{U}_{p}=F_{p}-\sum_{p^{\prime} \neq p, p^{\prime}=1}^{P} g_{p p^{\prime}} h_{p^{\prime}} c_{p^{\prime}} N_{p^{\prime}} \mathcal{U}_{p^{\prime}}\left|\Delta_{p^{\prime}}\right|, \quad 1 \leq p \leq P
$$

We have assumed that

$$
h_{m^{\prime}}=h_{p^{\prime}}[1+o(1)], \quad c_{m^{\prime}}=c_{p^{\prime}}[1+o(1)], \quad \mathcal{U}_{m^{\prime}}=\mathcal{U}_{p^{\prime}}[1+o(1)], a \rightarrow 0,
$$

for $x_{m^{\prime}} \in \Delta_{p^{\prime}}$. This assumption is justified if the functions $h(x), \mathcal{U}(x, \lambda)$,

$$
c(x)=\lim _{x_{m^{\prime}} \in \Delta_{x}, a \rightarrow 0} \frac{\left|S_{m^{\prime}}\right|}{a^{2}},
$$


and $N(x)$ are continuous. The function $h(x)$ and $N(x)$ are continuous by the assumption. The continuity of the $\mathcal{U}(x, \lambda)$ is proved in Section 3, and the continuity of $c(x)$ is assumed. If all the small bodies are identical, then $c(x)=c=$ const.

The sum in the right-hand side of (2.22) is the Riemannian sum for the integral

$$
\lim _{a \rightarrow 0} \sum_{p^{\prime}=1, p^{\prime} \neq p}^{P} g_{p p^{\prime}} h_{p^{\prime}} c_{p^{\prime}} N\left(x_{p^{\prime}}\right) \mathcal{U}_{p^{\prime}}=\int_{\mathcal{D}} g(x, y) h(y) c(y) N(y) \mathcal{U}(y, \lambda) d y
$$

Therefore, linear algebraic system (2.22) is a collocation method for solving integral equation

$$
\mathcal{U}(x, \lambda)=F(x, \lambda)-\int_{\mathcal{D}} g(x, y) h(y) c(y) N(y) \mathcal{U}(y, \lambda) d y
$$

Convergence of this method for solving equations with weakly singular kernels is proved in [8]. Applying the operator $-\nabla^{2}+\lambda$ and then taking the inverse Laplace transform of (2.25) yields

$$
u_{t}=\Delta u+f(x)-q(x) u, q(x):=h(x) c(x) N(x) .
$$

One concludes that the limiting equation for the temperature contains the term $q(x) u$. Thus, the embedding of many small particles creates a distribution of source and sink terms in the medium, the distribution of which is described by the term $q(x) u$.

If one solves equation $(2.25)$ for $\mathcal{U}(x, \lambda)$, or linear algebraic system $(2.22)$ for $\mathcal{U}_{p}(\lambda)$, then one can Laplaceinvert $\mathcal{U}(x, \lambda)$ for $\mathcal{U}(x, t)$. Numerical methods for Laplace inversion from the real axis are discussed in [9] - [10].

If one is interested only in the average temperature, one can use the relation

$$
\lim _{T \rightarrow \infty} \frac{1}{T} \int_{0}^{T} u(x, t) d t=\lim _{\lambda \rightarrow 0} \lambda \mathcal{U}(x, \lambda):=\psi(x)
$$

Relation (2.27) is proved in Section 3, which holds if the limit on one of its sides exists. The limit on the right-hand side of (2.27) can be calculated by the formula

$$
\psi(x)=(I+B)^{-1} \varphi, \varphi=\int \frac{1}{4 \pi|x-y|} f(y) d y .
$$

Here, $B$ is the operator

$$
B \psi:=\int \frac{q(y) \psi(y)}{4 \pi|x-y|} d y, \quad q(x):=h(x) c(x) N(x) .
$$

From the physical point of view the function $h(x)$ is non-positive because the flux $-\nabla u$ of the heat flow is proportional to the temperature $u$ and is directed along the outer normal $N$ : $-u_{N}=h_{1} u$, where $h_{1}=-h>0$. Thus, $q \leq 0$. It is proved in [11] - [12] that zero is not an eigenvalue of the operator $-\nabla^{2}+q(x)$ provided that $q(x) \geq 0$ and $q=O\left(\frac{1}{|x|^{2+\epsilon}}\right)$ as $|x| \rightarrow \infty, \epsilon>0$. In our case, $q(x)=0$ outside $\mathcal{D}$, so the operator $(I+B)^{-1}$ exists and is bounded in $C(\mathcal{D})$. Let us formulate the basic result we have proved.

Theorem 2.1. Assume (1.1), (2.11), and $h \leq 0$. Then, there exists the limit $\mathcal{U}(x, \lambda)$ of $\mathcal{U}_{e}(x, \lambda)$ as $a \rightarrow 0, \mathcal{U}(x, \lambda)$ solves equation (2.25), and there exists the limit (2.27), where $\psi(x)$ is given by formula (2.28). 


\section{Proofs of some lemmas}

Lemma 3.1. Assume (2.11). Then relation (2.10) holds.

Proof. One has

$$
\begin{gathered}
\mathcal{J}_{1, m}:=\left|g\left(x, x_{m}\right) Q\right|=O\left(\frac{\left|Q_{m}\right| e^{-\sqrt{x}\left|x-x_{m}\right|}}{4 \pi\left|x-x_{m}\right|}\right) \leq \frac{e^{-1} \mid Q_{m}}{\left|x-x_{m}\right|},\left|x-x_{m}\right| \geq d . \\
\mathcal{J}_{2, m}:=\int_{\mathcal{S}_{m}} \frac{e^{-\sqrt{\lambda}\left|x-x_{m}\right|}}{4 \pi\left|x-x_{m}\right|} \max \left(\sqrt{\lambda} a, \frac{a}{\left|x-x_{m}\right|}\right)\left|\sigma_{m}\left(s^{\prime}\right)\right| d s^{\prime} \leq O\left(\frac{\left|Q_{m}\right| a}{\left|x-x_{m}\right|^{2}}\right),
\end{gathered}
$$

where $\left|x-x_{m}\right| \geq 2$, and the inequality

$$
\max _{\lambda \geq 0}\left(\sqrt{\lambda} e^{-\sqrt{\lambda}\left|x-x_{m}\right|}\right) \leq \frac{e^{-1}}{\left|x-x_{m}\right|}
$$

was used. The $\left|Q_{m}\right| \neq 0$. In fact, $\sigma_{m}$ keeps sign on $\mathcal{S}_{m}$, as follows from equation (2.5) as $a \rightarrow 0$. It follows from (3.1) - (3.2) that

$$
\left|\frac{\mathcal{J}_{2, m}}{\mathcal{J}_{1, m}}\right| \leq O\left(\left|\frac{a}{x-x_{m}}\right|\right) \leq O\left(\frac{a}{d}\right)<<1 .
$$

From (3.3) by the arguments similar to the given in [13] one obtains (2.10).

Lemma 3.2. Relation (2.14) holds.

Proof. Let us justify relation (2.14). As $a \rightarrow 0$, one has

$$
\frac{\partial}{\partial N_{s}} \frac{e^{-\sqrt{\lambda}\left|s-s^{\prime}\right|}}{4 \pi\left|s-s^{\prime}\right|}=\frac{\partial}{\partial N_{s}} \frac{1}{4 \pi\left|s-s^{\prime}\right|}+\frac{\partial}{\partial N_{s}} \frac{e^{-\sqrt{\lambda}\left|s-s^{\prime}\right|}-1}{4 \pi\left|s-s^{\prime}\right|} .
$$

It is known (see [5]) that

$$
\int_{\mathcal{S}_{m}} d s \int_{\mathcal{S}_{m}} \frac{\partial}{\partial N_{s}} \frac{1}{4 \pi\left|s-s^{\prime}\right|} \sigma_{m}\left(s^{\prime}\right) d s^{\prime}=-\int_{\mathcal{S}_{m}} \sigma_{m}\left(s^{\prime}\right) d s^{\prime}=-Q_{m} .
$$

On the other hand, as $a \rightarrow 0$, one has

$$
\left|\int_{\mathcal{S}_{m}} d s \int_{\mathcal{S}_{m}} \frac{e^{-\sqrt{\lambda}\left|s-s^{\prime}\right|}-1}{4 \pi\left|s-s^{\prime}\right|} \sigma_{m}\left(s^{\prime}\right) d s^{\prime}\right| \leq\left|Q_{m}\right| \int_{\mathcal{S}_{m}} d s \frac{1-e^{-\sqrt{\lambda}\left|s-s^{\prime}\right|}}{4 \pi\left|s-s^{\prime}\right|}=o\left(Q_{m}\right) .
$$

The relations (3.5) and (3.6) justify (2.14).

Lemma 3.3. Relation (2.27) holds.

Proof. Denote

$$
\frac{1}{t} \int_{0}^{t} u(t) d t:=v(t), \quad \bar{u}(\sigma):=\int_{0}^{\infty} e^{-\sigma t} u(t) d t
$$

Then

$$
\bar{v}(\lambda)=\int_{\lambda}^{\infty} \frac{\bar{u}(\sigma)}{\sigma} d \sigma
$$

by the properties of the Laplace transform. Assume that the limit $v(\infty):=v_{\infty}$ exists:

$$
\lim _{t \rightarrow \infty} v(t)=v_{\infty}
$$


Then,

Indeed,

$$
v_{\infty}=\lim _{\lambda \rightarrow 0} \lambda \int_{0}^{\infty} e^{-\lambda t} v(t) d t=\lim _{\lambda \rightarrow 0} \lambda \bar{v}(\lambda) .
$$

$$
\lambda \int_{0}^{\infty} e^{-\lambda t} d t=1
$$

so

$$
\lim _{\lambda \rightarrow 0} \lambda \int_{0}^{\infty} e^{-\lambda t}\left(v(t)-v_{\infty}\right) d t=0
$$

and (3.7) is verified. One has

$$
\lim _{\lambda \rightarrow 0} \lambda \bar{v}(\lambda)=\lim _{\lambda \rightarrow 0} \int_{\lambda}^{\infty} \frac{\lambda}{\sigma} \bar{u}(\sigma) d \sigma=\lim _{\lambda \rightarrow 0} \lambda \bar{u}(\lambda) .
$$

Let us check this:

$$
\lim _{\lambda \rightarrow 0} \int_{\lambda}^{\infty} \frac{\lambda}{\sigma} \bar{u}(\sigma) d \sigma=\lim _{\lambda \rightarrow 0} \int_{\lambda}^{\infty} \frac{\lambda}{\sigma^{2}} \sigma \bar{u}(\sigma) d \sigma=\lim _{\sigma \rightarrow 0} \sigma \bar{u}(\sigma),
$$

where we have used the relation $\int_{\lambda}^{\infty} \frac{\lambda}{\sigma^{2}} d \sigma=1$.

Alternatively, let $\sigma^{-1}=\gamma$. Then,

$$
\int_{\lambda}^{\infty} \frac{\lambda}{\sigma^{2}} \sigma \bar{u}(\sigma) d \sigma=\frac{1}{1 / \lambda} \int_{0}^{1 / \lambda} \frac{1}{\gamma} \bar{u}\left(\frac{1}{\gamma}\right) d \gamma=\frac{1}{\omega} \int_{0}^{\omega} \frac{1}{\gamma} \bar{u}\left(\frac{1}{\gamma}\right) d \gamma .
$$

If $\lambda \rightarrow 0$, then $\omega=\lambda^{-1} \rightarrow \infty$, and if $\psi:=\gamma^{-1} \bar{u}\left(\gamma^{-1}\right)$, then

$$
\lim _{\omega \rightarrow \infty} \frac{1}{\omega} \int_{0}^{\omega} \psi d \gamma=\psi(\infty)=\lim _{\gamma \rightarrow \infty} \gamma^{-1} \bar{u}\left(\gamma^{-1}\right)=\lim _{\sigma \rightarrow 0} \sigma \bar{u}(\sigma)
$$

\section{References}

[1] V. Jikov, S. Kozlov, O. Oleinik. Homogenization of differential operators and integral functionals. Springer, Berlin, 1994.

[2] V. Marchenko, E. Khruslov. Homogenization of partial differential equations. Birkhäuser, Boston, 2006.

[3] D. Gioranescu, P. Donato. An introduction to homogenization. Oxford Univ. Press, Oxford, 1999.

[4] A. Bensoussan, J.-L.Lions, G. Papanicolau. Asymptotic analysis for periodic structures. AMS Chelsea Publishing, Providence, RI, 2011.

[5] A. G. Ramm. Wave scattering by small bodies of arbitrary shapes. World Sci. Publishers, Singapore, 2005.

[6] A.G.Ramm. Inverse problems. Springer, New York, 2005.

[7] A.G.Ramm. Wave scattering by many small bodies and creating materials with a desired refraction coefficient. Afrika Matematika, 22, No. 1, (2011), 33-55.

[8] A.G.Ramm. A collocation method for solving integral equations. Internat. Journ. Comp. Sci and Math., 3, No. 2, (2009), 222-228.

[9] A.G.Ramm. Inversion of the Laplace transform from the real axis. Inverse problems, 2, (1986), L55-59.

[10] A.G.Ramm, S.Indratno. Inversion of the Laplace transform from the real axis using an adaptive iterative method. Internat. Jour. Math. Math. Sci (IJMMS). Vol. 2009, Article 898195, 38 pages.

[11] A.G.Ramm. Sufficient conditions for zero not to be an eigenvalue of the Schrödinger operator. J. Math. Phys., 28, (1987), 1341-1343.

[12] A.G.Ramm. Conditions for zero not to be an eigenvalue of the Schrödinger operator. J. Math. Phys. 29, (1988), 1431-1432.

[13] A.G.Ramm. Many-body wave scattering by small bodies and applications. J. Math. Phys., 48, No. 10, (2007), 103511.

[14] A.G.Ramm. Wave scattering by many small particles embedded in a medium. Phys. Lett. A, 372/17, (2008), 3064-3070.

[15] A.G.Ramm. A method for creating materials with a desired refraction coefficient. Internat. Journ. Mod. Phys B, 24, No. 27, (2010), 5261-5268.

[16] A.G.Ramm. Materials with a desired refraction coefficient can be created by embedding small particles into a given material. International Journal of Structural Changes in Solids (IJSCS), 2, No. 2, (2010), 17-23.

[17] A.G.Ramm. Distribution of particles which produces a "smart" material. Jour. Stat. Phys., 127, No. 5, (2007), 915-934.

[18] A.G.Ramm. Scattering of scalar waves by many small particles. AIP Advances, 1, (2011), 022135.

[19] A.G.Ramm. Scattering of electromagnetic waves by many small cylinders. Results in Physics, 1, No. 1, (2011), 13-16. 